\title{
Raman-scattering study of the InGaN alloy over the whole composition range
}

\author{
S. Hernández \\ Department of Physics, University of Stralhclyde, Glasgow, G4 ONG, Scotland, United Kingdom \\ R. Cuscó, D. Pastor, and L. Artús ${ }^{a)}$ \\ Institut Jaume Almera, Consell Superior d'Investigacions Científiques (CSIC), Lluís Solé i Sabarís s.n., \\ 08028 Barcelona, Spain \\ K. P. O'Donnell and R. W. Martin \\ Department of Physics, University of Strathclyde, Glasgow, G4 ONG, Scotland, United Kingdom \\ I. M. Watson \\ Institute of Photonics, University of Strathclyde, Glasgow G4 ONW, Scotland, United Kingdom \\ Y. Nanishi \\ Department of Photonics, Ritsumeikan University, 1-1-1 Noji-higashi, Kusatsu 525-8577, Japan \\ E. Calleja \\ Instituto de Sistemas Optoelectrónicos y Microtecnologia (ISOM) and Departamento Ingeniería Electrónica, \\ Escuela Técnica Superior de Ingenieros de (ETSI) Telecomunicación, Universidad Politécnica \\ de Madrid, Ciudad Universitaria, 28040 Madrid, Spain
}

(Received 11 March 2005; accepted 2 May 2005; published online 5 July 2005)

\begin{abstract}
We present Raman-scattering measurements on $\operatorname{In}_{x} \mathrm{Ga}_{1-x} \mathrm{~N}$ over the entire composition range of the alloy. The frequencies of the $A_{1}(\mathrm{LO})$ and $E_{2}$ modes are reported and show a good agreement with the one-mode behavior dispersion predicted by the modified random-element isodisplacement model. The $A_{1}(\mathrm{LO})$ mode displays a high intensity relative to the $E_{2}$ mode due to resonant enhancement. For above band-gap excitation, the $A_{1}(\mathrm{LO})$ peak displays frequency shifts as a function of the excitation energy due to selective excitation of regions with different In contents, and strong multiphonon scattering up to 3LO is observed in outgoing resonance conditions. (C) 2005 American Institute of Physics. [DOI: 10.1063/1.1940139]
\end{abstract}

\section{INTRODUCTION}

In recent years the InGaN alloy has attracted a great deal of interest because of its successful application in the realization of high-quality light-emitting diodes covering the green-to-violet spectral range. ${ }^{1}$ However, the large difference in interatomic spacing between $\mathrm{GaN}$ and $\mathrm{InN}$ makes the growth of InGaN alloys challenging, as it gives rise to compositional inhomogeneity and strains that strongly affect the optical properties of the InGaN layers. Thus, variations observed in the photoluminescence (PL) emission energy and in the longitudinal-optical (LO) Raman peak frequency with the excitation energy have been ascribed to selective resonant excitation of regions with different In contents. ${ }^{2,3}$ On the other hand, structural studies of InGaN epilayers have revealed strain and compositional gradients along the growth direction, ${ }^{4}$ and an alternative explanation of the PL and LOphonon dependence on the excitation energy in terms of differently strained regions has been suggested. ${ }^{5}$

Raman scattering is a standard technique to characterize the strain and the composition in III-V alloy systems. ${ }^{6,7}$ However, to date a poor knowledge of the long-wavelength optical-phonon behavior in the InGaN exists. In a ternary semiconductor $A_{x} B_{1-x} C$, the optical phonons may exhibit one-mode or two-mode behavior. One-mode alloys only

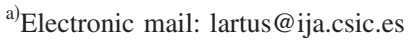

present one set of LO and TO phonons, with frequencies which show an almost linear dependence from one end member $(A C)$ to the other $(B C)$. Two-mode behavior alloys exhibit two sets of LO and TO phonons whose frequencies vary continuously from those of one end-member binary $(A C, B C)$ to the impurity mode in the other end-member binary ( $B C: A$ and $A C: B$, respectively). Most of the Ramanscattering studies of phonons in InGaN alloys have focused on the low-In composition range ${ }^{5,8,9}$ because of the difficulties in growing samples with higher In content. Extrapolating the observed frequency dependence for $x<0.5$ to the $\mathrm{InN}$ $(x=1)$ end, Alexson et al. ${ }^{9}$ concluded that the $A_{1}(\mathrm{LO})$ mode follows a one-mode behavior, whereas they suggested a twomode behavior for the $E_{2}$ mode based on its departure from linear dependence. On the other hand, theoretical calculations based on the modified random-element ISO displacement ${ }^{10}$ (MREI) model predict one-mode behavior for both $A_{1}(\mathrm{LO})$ and $E_{2}$ modes.

In this work, we report a study of the $A_{1}(\mathrm{LO})$ and $E_{2}$ optical phonons of $\operatorname{In}_{x} \mathrm{Ga}_{1-x} \mathrm{~N}$ over the entire composition range of the alloy. The study has been performed on epitaxial layers with 13 different compositions that were grown either by metal-organic chemical-vapor deposition (MOCVD) or by molecular-beam epitaxy (MBE) under different conditions. We find one-mode behavior for both $A_{1}(\mathrm{LO})$ and $E_{2}$ modes, and a good agreement with the frequencies given by 
the MREI calculations for all In compositions. Selective resonance effects as well as strain gradient effects on the $A_{1}$ (LO) frequency shifts are discussed. The resonant excitation is clearly evidenced by the observation of a strong $A_{1}(\mathrm{LO})$ peak and multiphonon peaks up to $3 A_{1}(\mathrm{LO})$.

\section{EXPERIMENT}

The $\mathrm{In}_{x} \mathrm{Ga}_{1-x} \mathrm{~N}$ layers used in these experiments were all grown, either by MOCVD or MBE, on $\mathrm{Al}_{2} \mathrm{O}_{3}$ (0001) substrates with a $\mathrm{GaN}$ or InN buffer layer. Details of the growth conditions are given in Ref. 11 for the MOCVD samples, and in Refs. 12 and 13 for the MBE samples. MOCVD epilayers with In composition of $x=0.06,0.10,0.16,0.20$, and 0.24 , and MBE epilayers with $x=0.20,0.24,0.27,0.37,0.58,0.60$, 0.78 , and 0.96 were studied. The In composition of the layers was determined by wavelength dispersive x-ray (WDX) spectroscopy measurements using a Cameca SX100 analyzer as described in Ref. 14, except for the MBE epilayers with $x \leqslant 0.37$, which were characterized by means of $\mathrm{x}$-ray diffraction measurements. ${ }^{12}$ The thickness of the $\operatorname{In}_{x} \mathrm{Ga}_{1-x} \mathrm{~N}$ epilayers was between 220 and $300 \mathrm{~nm}$, which is above the critical layer thickness for strain relaxation. ${ }^{15}$

The Raman-scattering spectra were recorded at RT in backscattering configuration using a Jobin-Yvon T64000 spectrometer equipped with a $\mathrm{LN}_{2}$-cooled charge-coupled device (CCD). To minimize Raman-scattering signal from the buffer layer we used the 457.9-nm line of an $\mathrm{Ar}^{+}$laser, which corresponds to above-band-gap excitation for most of the $\mathrm{In}_{x} \mathrm{Ga}_{1-x} \mathrm{~N}$ epilayers. From the available opticalabsorption coefficients ${ }^{16,17}$ we estimate that the $1 / 2 \alpha$ penetration depth for this wavelength is less than $55 \mathrm{~nm}$ for all the InGaN epilayers with $x>0.23$. This ensures that most of the Raman-scattering signal comes from the near surface region, thus avoiding contributions from the substrate and from regions close to the InGaN-substrate interface that could be strained. For the layers with the lowest In fraction $(x=0.06$ and $x=0.10$ ), the $457.9-\mathrm{nm}$ excitation is in the transparency region and therefore, we used instead the $325-\mathrm{nm}$ line of a $\mathrm{He}-\mathrm{Cd}$ laser as an excitation source to ensure that only a superficial layer was probed by the Raman-scattering measurements. Additional measurements using several $\mathrm{Ar}^{+}$lines were performed on samples with $x=0.27$ to explore the nearresonance region.

\section{RESULTS AND DISCUSSION}

\section{A. Phonon mode dispersion with alloy composition}

Figure 1 shows the room-temperature Raman spectra of a set of wurtzite $\operatorname{In}_{x} \mathrm{Ga}_{1-x} \mathrm{~N}$ epilayers with In fractions ranging from $x=0.06$ up to 0.97 recorded in the $z(x x) \bar{z}$ backscattering configuration. In this configuration, only the $E_{2}$ and the $A_{1}(\mathrm{LO})$ modes are allowed. For clarity, the spectra corresponding to different In compositions have been scaled to exhibit similar intensities and the luminescence background has been subtracted from the spectrum of $x=0.16$. As can be seen in Fig. 1, the Raman spectra display only one $A_{1}(\mathrm{LO})$ and one $E_{2}$ peak over the entire composition range of the alloy. This confirms the theoretical predictions of the one-mode-type behavior of optical phonons in the $\operatorname{In}_{x} \mathrm{Ga}_{1-x} \mathrm{~N}$

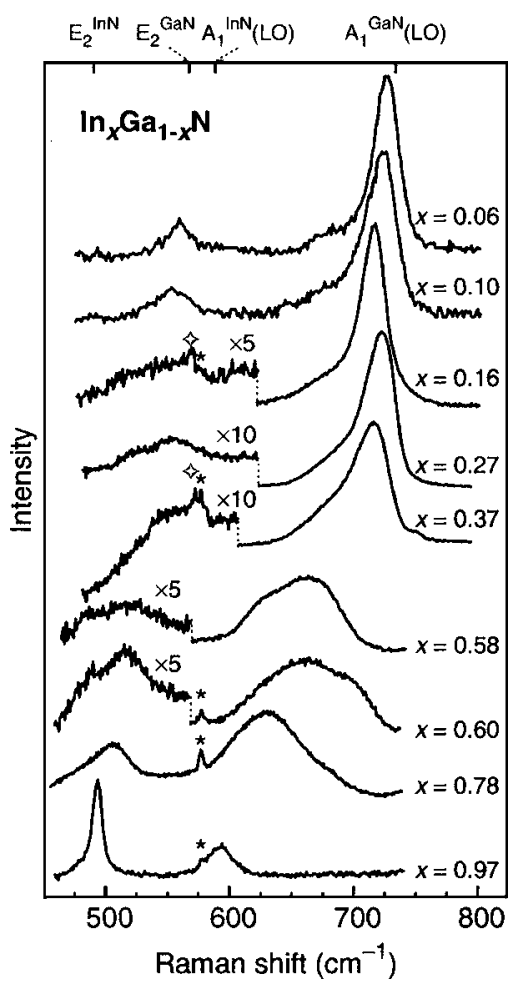

FIG. 1. Room-temperature Raman spectra of InGaN epilayers for nine different In fractions covering the entire alloy range. The Raman spectra were excited with the 457.9-nm line of an $\mathrm{Ar}^{+}$laser except for the samples with $x=0.06$ and $x=0.10$, which were excited with the 325-nm line of a $\mathrm{He}-\mathrm{Cd}$ laser. The peaks marked with diamonds and asterisks are due to the modes of the $\mathrm{GaN}$ buffer and of the $\mathrm{Al}_{2} \mathrm{O}_{3}$ substrate, respectively.

alloy. ${ }^{10,18}$ While the alloys with compositions close to the end-member compounds show well-defined Raman peaks, a significant broadening can be seen for intermediate compositions, particularly for the $E_{2}$ mode. The peak broadening reflects the increased lattice disorder and composition fluctuations in the alloy at intermediate In composition. This can be clearly seen in Fig. 2 where we have plotted the peak frequency and, as error bars, the width at half height of the $E_{2}$ and $A_{1}(\mathrm{LO})$ Raman peaks.

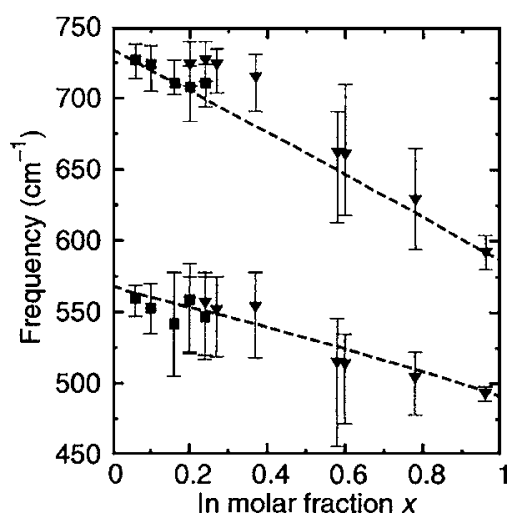

FIG. 2. Observed frequency for the $A_{1}(\mathrm{LO})$ and $E_{2}$ modes of the InGaN alloy vs In molar fraction for the 13 samples with different In compositions studied in the present work. The triangles correspond to MBE-grown samples, whereas the squares correspond to MOCVD-grown samples. The bars represent the width at half height of the Raman peaks. The composition dependency of the $A_{1}(\mathrm{LO})$ and $E_{2}$ mode energies predicted by the modified random-element isodisplacement model (Ref. 10) are plotted as dashed lines. 
The widths of the Raman lines in the alloy with $x$ $=0.97$ are close to those observed in pure $\mathrm{InN},{ }^{19}$ which indicates the high crystalline quality of the sample. In this sample, the intensity of the $A_{1}(\mathrm{LO})$ mode is much weaker than that of the $E_{2}$ mode. This could be due to the high density of free carriers in the epilayer, which was found to be $2.7 \times 10^{19} \mathrm{~cm}^{-3}$ by Hall measurements. Since the free-carrier excitations couple to the polar $A_{1}(\mathrm{LO})$ mode giving rise to phonon-plasmon modes, the Raman peak observed at the frequency of the bare $A_{1}(\mathrm{LO})$ mode comes only from the surface depletion region. ${ }^{20,21}$ This interpretation is supported by the observation in this sample of a Raman peak at $443 \mathrm{~cm}^{-1}$ (not shown in Fig. 1) that was previously reported in $n$-type InN epilayers and assigned to the $L^{-}$LO-plasmon coupled mode. ${ }^{19}$ When the In fraction is reduced in the In-rich alloys the width of both the $E_{2}$ and the $A_{1}(\mathrm{LO})$ peaks dramatically increases and the intensity of the $A_{1}(\mathrm{LO})$ mode becomes dominant. The overdamped nature of the free-carrier excitations in these highly disordered alloys could explain the absence of coupled modes. In fact, the $L^{-}$Raman peak close to the $A_{1}(\mathrm{TO})$ frequency can only be detected in the sample with $x=0.97$.

The $A_{1}(\mathrm{LO})$ mode develops a high-frequency shoulder in the sample with $x=0.60$. Such shoulders have been previously related to compositional inhomogeneities and spinodal decomposition, ${ }^{9}$ and to strain gradients over depth. ${ }^{22} \mathrm{We}$ do not observe this shoulder when the Raman spectrum is excited with the 514.5-nm line, which indicates that it is most likely due to compositional inhomogeneities leading to selective resonant excitation of regions with lower In fraction. For the samples with $x=0.60$ and $x=0.58$, a small feature can be observed on the low-frequency side of the $E_{2}$ peak that is due to the $E_{2}$ mode of the very thin low-temperature InN buffer layer that was deposited on the sapphire substrate prior to the $\mathrm{In}_{x} \mathrm{Ga}_{1-x} \mathrm{~N}$ growth.

Using the dependence on composition of the $\mathrm{InGaN}$ band gap given in Ref. 23 and the $A_{1}(\mathrm{LO})$ frequency dispersion as calculated in Ref. 10, we estimate that for the 2.71-eV excitation used in our Raman measurements an alloy with $x=0.17$ would be on the peak of the $A_{1}(\mathrm{LO})$ resonance profile. This agrees well with the Raman spectra displayed in Fig. 1, where the highest background luminescence and $A_{1}(\mathrm{LO}) / E_{2}$ intensity ratio were observed for the sample with $x=0.16$. Although the spectra of the samples with $x$ $=0.06$ and $x=0.10$ were excited with the $3.81-\mathrm{eV}$ line, well above the direct band gap of the alloy, the $A_{1}(\mathrm{LO})$ intensity still shows some enhancement probably due to a broad resonant Raman profile. It should be noted that according to Ref. 9 , the $A_{1}(\mathrm{LO}) / E_{2}$ intensity ratio is reversed when the spectra are recorded with 5.08-eV excitation.

At the Ga-rich end, the $A_{1}(\mathrm{LO})$ peak exhibits a lowfrequency tail extending down to $\approx 650 \mathrm{~cm}^{-1}$, which coincides with a region with a high phonon density of states in $\mathrm{GaN}^{24}$ Therefore, this frequency tail can be assigned to disorder-activated modes which become Raman active due to the relaxation of wave-vector conservation caused by the presence of structural defects and the random cation distribution in the alloy. As can be seen in Fig. 1, the lowfrequency tail becomes stronger as the In fraction increases, indicating that the effect of random cation distribution plays a major role in the width of the $A_{1}(\mathrm{LO})$ mode.

In Fig. 2 the $A_{1}(\mathrm{LO})$ and $E_{2}$ frequencies obtained from the Raman spectra are compared with the MREI model calculations reported in Ref. 10. We find a good agreement with the theoretical predictions over the whole composition range. The $E_{2}$ data show a wider dispersion because of the uncertainty introduced by the weak Raman signal and the large width of the mode. The $A_{1}(\mathrm{LO})$ mode allows a more precise determination of its peak frequency, which follows closely the theoretical dependence, except for a group of four points between $x=0.20$ and $x=0.37$ that are clearly above the expected frequency. It should be noted that these points correspond to MBE samples. In the $0.16 \leqslant x \leqslant 0.24$ range we have the MOCVD data (filled squares) nearly overlapping in composition the MBE data (filled triangles), and we can see that whereas the $A_{1}(\mathrm{LO})$ frequency in MOCVD samples agrees very well with the nearly linear one-mode dependence, for MBE samples it is systematically higher. It has been recently found by means of extended x-ray-absorption fine-structure (EXAFS) measurements ${ }^{25}$ that for the some In content, the In-N bond lengths in MOCVD samples were longer than those in MBE-grown samples. These structural differences could explain the presence of a residual compressive strain in the MBE samples producing an upward frequency shift of the phonons. The effect tends to be smaller towards the Inrich end, as already observed by EXAFS. ${ }^{25}$

\section{B. Near-resonance behavior and multiphonon scattering}

As discussed in Sec. IIA, resonant effects give rise to the intensity enhancement displayed by the polar $A_{1}(\mathrm{LO})$ mode in the mid- to low-In composition alloys $(0.16 \leqslant x \leqslant 0.37)$. Beside the enhancement of the Raman intensity of the infrared-active modes, electron-phonon coupling via the Fröhlich interaction can produce, near resonance, very strong scattering by $2 \mathrm{LO}$ phonons and higher-order multiphonon scattering. ${ }^{26}$ For excitation above the band gap in the absorption continuum, resonant multiphonon Raman-scattering lines were observed in the photoluminescence of doped $\mathrm{GaN}$ (Ref. 27) and resonant Raman scattering by $n$-LO modes up to $n=7$ were reported for Be- and C-implanted $\mathrm{GaN}^{28}$

To study the resonant behavior of InGaN, we have chosen the alloy composition $x=0.27$, which according to the band-gap dependence on composition given in Ref. 23, corresponds to a band gap of about $2.42 \mathrm{eV}$. Thus, excitation with the 2.41-eV line of the $\mathrm{Ar}^{+}$laser is very close to the direct band-gap resonance. Since the $A_{1}(\mathrm{LO})$ energy for this sample was determined to be $90 \mathrm{meV}$ from the Raman measurements, excitation with the 2.54-, 2.60-, and 2.71-eV lines of the $\mathrm{Ar}^{+}$laser lies close to the 1-, 2-, and 3LO outgoing resonances, respectively. In Fig. 3 we show the Raman spectra of the $n-A_{1}(\mathrm{LO})$ modes for $n=1,2,3$ obtained from the $\mathrm{In}_{0.27} \mathrm{Ga}_{0.73} \mathrm{~N}$ sample using these excitation lines. Note that the Raman spectra have been normalized to the intensity of the $A_{1}(\mathrm{LO})$ mode. The actual intensity of the $A_{1}(\mathrm{LO})$ mode 


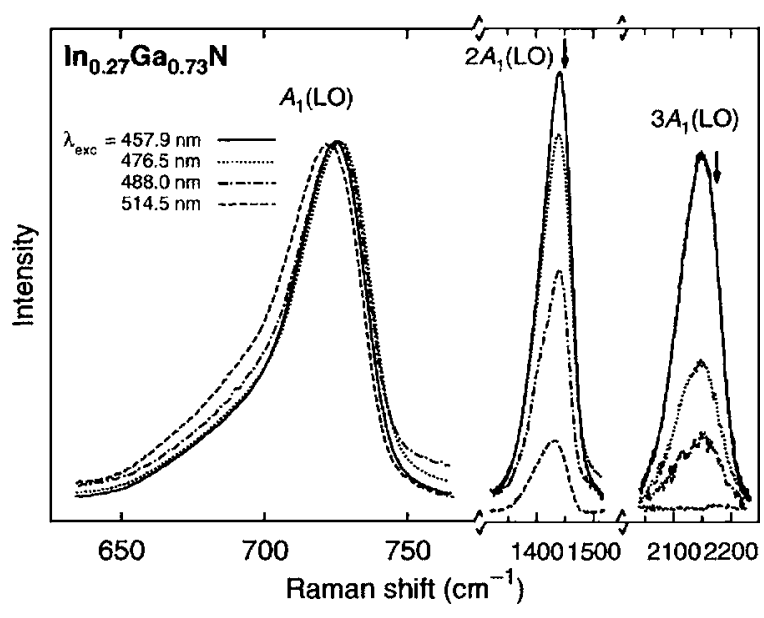

FIG. 3. Multiple $A_{1}(\mathrm{LO})$-phonon peaks observed under near-resonant conditions for different excitation lines. To allow comparison between relative intensities of the $n$-LO-phonon modes, the Raman spectra have been normalized to the intensity of the $A_{1}(\mathrm{LO})$ mode and the luminescence background has been subtracted. The arrows indicate the corresponding multiples of the zone-center $A_{1}(\mathrm{LO})$ frequency.

in the spectrum excited with the 2.41-eV line was about 40 times higher than in the spectrum excited with the $2.71-\mathrm{eV}$ line.

The $A_{1}(\mathrm{LO})$ peak shifts to lower frequencies by about $4 \mathrm{~cm}^{-1}$ and broadens asymmetrically towards low frequencies when the excitation energy is reduced from 2.71 to $2.41 \mathrm{eV}$. This behavior cannot be explained by strain gradients along the growth direction reported in InGaN alloys, because the longer excitation wavelengths are less absorbed and therefore, probe a deeper region where the sample is more strained. ${ }^{22}$ This effect would lead to highfrequency shifts of the phonons excited with the $2.41-\mathrm{eV}$ line, contrary to the experimental observation. On the other hand, frequency shifts in the $A_{1}(\mathrm{LO})$ Raman peak can be attributed to selective resonant excitation of regions with different In contents. ${ }^{2,3}$ For lower excitation energies, regions with higher In fraction will have a matching band gap. According to Ref. 23, an In fraction of $x=0.27$ yields a band gap of $2.42 \mathrm{eV}$, whereas $E_{G}=2.71$ occurs at $x=0.19$. Higher In composition yields lower phonon frequencies, which is consistent with the observed downshift of the $A_{1}(\mathrm{LO})$ mode in the spectra excited with the 2.41-eV line. However, the magnitude of the observed downward shift $\left(\approx 4 \mathrm{~cm}^{-1}\right)$ is smaller than the shift expected for an increase of In fraction from $x=0.19$ to $x=0.27$, which according to Ref. 10 is $\approx 13 \mathrm{~cm}^{-1}$. This suggests that the selective resonance shift is partly compensated by the strain gradient effect discussed above.

We note that a substantial $A_{1}(\mathrm{LO})$ asymmetrical broadening is observed for the lower excitation energies; the full width at half height increases from $33 \mathrm{~cm}^{-1}$ at $2.71 \mathrm{eV}$ up to $62 \mathrm{~cm}^{-1}$ at $2.41 \mathrm{eV}$. Such a broadening cannot be attributed to disorder in the deeper region probed by the $2.41-\mathrm{eV}$ excitation, since it was shown that the density of structural defects is higher in the upper part of the $\operatorname{In}_{x} \mathrm{Ga}_{1-x} \mathrm{~N}$ films. ${ }^{22}$ This broadening actually reflects the distribution of regions with different In fractions in the scattering volume, and therefore the asymmetric line shape of the $A_{1}(\mathrm{LO})$ mode cannot be accounted for solely in terms of phonon correlation length, as suggested in previous works. ${ }^{8}$

The Raman spectrum excited with the 2.41-eV line displays a weak $2 \mathrm{LO}$ peak, but no signal in the $3 \mathrm{LO}$ region could be detected. The relative intensity of the $2 \mathrm{LO}$ peak increases substantially under excitation with the 2.54-eV line and a $3 \mathrm{LO}$ feature begins to be visible. By increasing the excitation energy to $2.60 \mathrm{eV}$, which as mentioned above is close to the $2 \mathrm{LO}$ outgoing resonance in this sample, 2LO scattering becomes as intense as first-order scattering. A strong enhancement of 2LO scattering near the outgoing resonance is characteristic of an iteration of the intraband Fröhlich interaction via excitonic intermediate states. ${ }^{29}$ TwoLO-phonon scattering is mainly governed by near zonecenter phonons because of the $q^{-1}$ dependence of the Fröhlich interaction. Thus, it gives rise to sharp peaks at about twice the frequency of the first-order LO peak. We observe a $2 \mathrm{LO}$ peak which is centered about $10 \mathrm{~cm}^{-1}$ below the exact multiple of the first-order frequency (arrow) and exhibits a slight asymmetry with a low-frequency tail. Both features could result from the combined effect of the participation of $q \neq 0$ phonons and the distribution of different In fractions in the film. Even though the $3 \mathrm{LO}$ intensity is higher with the $2.60-\mathrm{eV}$ excitation than with the 2.54-eV excitation, the $3 \mathrm{LO} / 2 \mathrm{LO}$ intensity ratio is roughly the same. By contrast, excitation with the $2.71-\mathrm{eV}$ line greatly enhances the $3 \mathrm{LO}$ peak, which becomes comparable in intensity with the $2 \mathrm{LO}$ peak. The 2.71-eV line is close to the outgoing 3LO resonance. Then, resonant Raman scattering via real intermediate exciton states in a cascade process ${ }^{30}$ becomes possible, which contributes to the strong intensity observed for the 3LO peak. Since wave-vector conservation in the cascade model requires the emission of $q \neq 0 \mathrm{LO}$ phonons, the 3LO Raman peak contains important contributions from phonons away from the zone center. This is reflected in a peak frequency clearly below $3 \omega_{\mathrm{LO}}(\Gamma)$, as can be seen in Fig. 3. No Raman signal was detected in the 4LO frequency region due to the small probability of high-order scattering and the excitation energy being too low to excite a cascade process involving four LO phonons.

\section{CONCLUSION}

We have carried out a Raman-scattering study of the phonon behavior of the $\operatorname{In}_{x} \mathrm{Ga}_{1-x} \mathrm{~N}$ alloy over the whole composition range. The one-mode behavior of the $A_{1}(\mathrm{LO})$ and $E_{2}$ modes has been confirmed and the respective phonon frequencies have been determined for the whole composition range, showing good agreement with the previous MREI model calculations. We have found systematic frequency deviations in MBE-grown samples that we ascribe to local structural differences in bond length also detected by EXAFS measurements. A substantial broadening is observed for intermediate In fractions due to intrinsic disorder and composition fluctuations.

In most samples, the $A_{1}(\mathrm{LO})$ mode displays a very high intensity relative to the $E_{2}$ mode, which indicates a resonant enhancement via Fröhlich mechanisms of the polar $A_{1}(\mathrm{LO})$ 
mode. A more detailed study using above band-gap excitation for the sample with $x=0.27$ revealed a frequency shift of the $A_{1}(\mathrm{LO})$ mode with excitation wavelength that can be accounted for by the selective excitation of regions with different In contents. This effect has been shown to be partly compensated by the presence of strain gradients along the growth direction. Excitation in near outgoing resonance conditions allowed us to observe strong multiphonon peaks up to $3 \mathrm{LO}$.

\section{ACKNOWLEDGMENTS}

This work has been supported by the Spanish Ministry of Science and Technology under Contract No. MAT20011878. One of the authors (S.H.) acknowledges financial support from the Spanish Ministry of Education and Science.

${ }^{1}$ S. Nakamura, M. Senoh, N. Iwasa, S. Nagahama, T. Yamada, and T. Mukail, Jpn. J. Appl. Phys., Part 2 34, L1332 (1995).

${ }^{2}$ D. Behr, J. Wagner, A. Ramakrishnan, H. Obloh, and K.-H. Bachem, Appl. Phys. Lett. 73, 241 (1998).

${ }^{3}$ N. Wieser, O. Ambacher, H.-P. Felsl, L. Görgens, and M. Stutzmann, Appl. Phys. Lett. 74, 3981 (1999)

${ }^{4}$ S. Pereira, M. R. Correira, E. Pereira, K. P. O’Donnell, C. Trager-Cowan, F. Sweeney, and E. Alves, Phys. Rev. B 64, 205311 (2001).

${ }^{5}$ M. R. Correia, S. Pereira, E. Pereira, J. Frandon, and E. Alves, Appl. Phys. Lett. 83, 4761 (2003).

${ }^{6}$ L. Artús, R. A. Stradling, Y. B. Li, S. J. Webb, W. T. Yuen, S. J. Chung, and R. Cuscó, Phys. Rev. B 54, 16373 (1996).

${ }^{7}$ S. Hernández, N. Blanco, I. Mártil, G. González-Díaz, R. Cuscó, and L. Artús, J. Appl. Phys. 93, 9019 (2003).

${ }^{8}$ T. Sugiura, Y. Kawaguchi, T. Tsukamoto, H. Andoh, M. Yamaguchi, K.
Hiramatsu, and N. Sawaki, Jpn. J. Appl. Phys., Part 1 40, 5955 (2001).

${ }^{9}$ D. Alexson et al., J. Appl. Phys. 89, 798 (2001).

${ }^{10}$ H. Grille, C. Schnittler, and F. Bechstedt, Phys. Rev. B 61, 6091 (2000).

${ }^{11}$ C. J. Deatcher, C. Liu, S. Pereira, M. Lada, A. G. Cullis, Y. J. Sun, O. Brandt, and I. M. Watson, Semicond. Sci. Technol. 18, 212 (2003).

${ }^{12}$ F. B. Naranjo, S. Fernández, M. A. Sánchez-García, F. Calle, E. Calleja, A. Trampert, and K. H. Ploog, Mater. Sci. Eng., B 93, 131 (2002).

${ }^{13}$ M. Kurouchi, T. Araki, H. Naoi, T. Yamaguchi, A. Suzuki, and Y. Nanishi, Phys. Status Solidi B 241, 2843 (2004).

${ }^{14}$ R. W. Martin, P. R. Edwards, K. P. O’Donnell, E. G. Mackay, and I. M. Watson, Phys. Status Solidi A 192, 117 (2002).

${ }^{15}$ S. Pereira et al., Appl. Phys. Lett. 81, 1207 (2002).

${ }^{16}$ J. W. Trainor and K. Rose, J. Electron. Mater. 3, 821 (1974).

${ }^{17}$ L. Siozade, J. Leymarie, P. Disseix, A. Vasson, M. Mihailovic, N. Grandjean, M. Leroux, and J. Massies, Solid State Commun. 115, 575 (2000).

${ }^{18}$ S. Yu, K. W. Kim, L. Bergman, M. Dutta, M. A. Stroscio, and J. M. Zavada, Phys. Rev. B 58, 15283 (1998).

${ }^{19}$ V. Y. Davydov et al., Appl. Phys. Lett. 75, 3297 (1999).

${ }^{20}$ G. Abstreiter, M. Cardona, and A. Pinczuk, Light Scattering in Solids IV, Topics in Applied Physics Vol. 54 (Springer, Berlin, 1984).

${ }^{21}$ L. Artús, R. Cuscó, J. Ibáñez, N. Blanco, and G. González-Díaz, Phys. Rev. B 60, 5456 (1999).

${ }^{22}$ M. R. Correia et al., Appl. Phys. Lett. 85, 2235 (2004).

${ }^{23}$ J. Wu, W. Walukiewicz, K. M. Yu, J. W. Agen III, E. E. Haller, H. Lu, and W. J. Schaff, Appl. Phys. Lett. 80, 4741 (2002).

${ }^{24}$ V. Y. Davydov et al., Phys. Rev. B 58, 12899 (1998).

${ }^{25}$ V. Katchkanov et al., Mater. Res. Soc. Symp. Proc. 831, E3.30 (2004).

${ }^{26}$ M. Cardona, Light Scattering in Solids II, Topics in Applied Physics Vol. 50 (Springer, Berlin, 1982).

${ }^{27}$ D. J. Dewsnip et al., Semicond. Sci. Technol. 12, 55 (1997).

${ }^{28}$ W. H. Sun, S. J. Chua, L. S. Wang, and X. H. Zhang, J. Appl. Phys. 91, 4917 (2002).

${ }^{29}$ A. García-Cristóbal, A. Cantarero, C. Trallero-Giner, and M. Cardona, Phys. Rev. B 49, 13430 (1994).

${ }^{30}$ R. M. Martin and C. M. Varma, Phys. Rev. Lett. 26, 1241 (1971). 